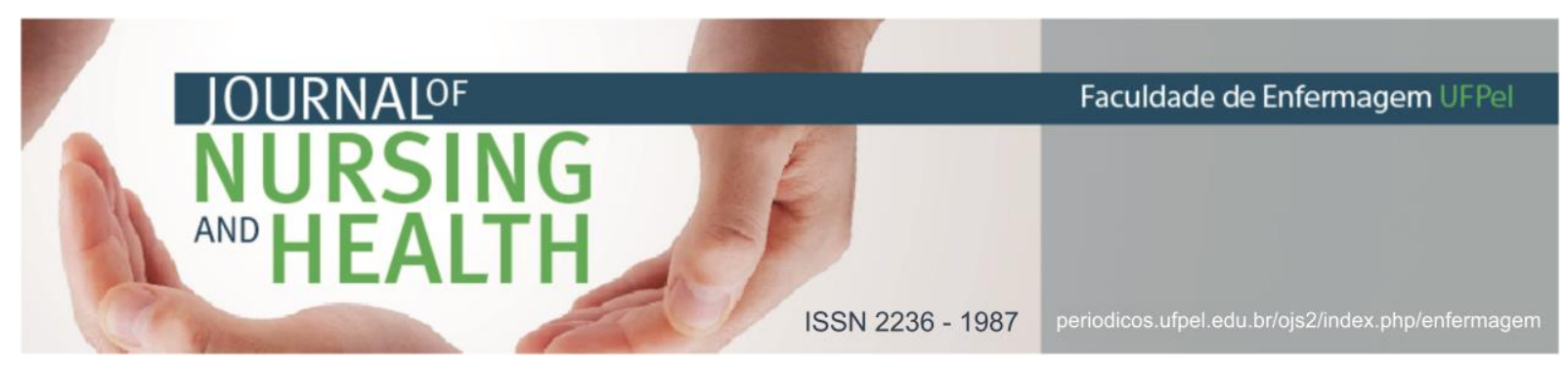

ARTIGO ORIGINAL

\title{
A alta dos usuários no centro de atenção psicossocial ${ }^{*}$
}

\author{
The discharge of the users in the psychosocial care center \\ El alta de los usuarios en el centro de atención psicosocial
}

Kantorski, Luciane Prado ${ }^{1}$; Guedes, Ariane da Cruz²; Machado, Roberta Antunes³; Couto, Maria Laura de Oliveira ${ }^{4}$; Santos, Vinícius Boldt dos $^{5}$; Pinheiro, Guilherme Emanuel Weiss ${ }^{6}$

Como citar este artigo: Kantorski LP, Guedes AC, Machado RA, Couto MLO, Santos VB, Pinheiro GEW. A alta dos usuários no centro de atenção psicossocial. J. nurs. health. 2019;9(3):e199305

\section{RESUMO}

Objetivo: caracterizar a alta dos usuários no Centro de Atenção Psicossocial. Método: estudo documental com abordagem quantitativa com dados do livro de registro de alta dos usuários, de 2006 a 2016. Foram analisadas as variáveis sexo, motivo de alta e diagnóstico. Resultados: a amostra constituiu 1.772 altas, distribuídas sem grande disparidade entre o sexo feminino (50,9\%) e masculino $(46,1 \%)$. A alta clínica é o motivo prevalente $(55,6 \%)$ nos 11 anos estudados e entre os três principais diagnósticos: transtorno afetivo bipolar $(62,1 \%)$, episódios de depressão $(59,2 \%)$ e esquizofrenia $(58,3 \%)$. A esquizofrenia foi o diagnóstico prevalente entre os usuários $(24,2 \%)$, seguida pelo transtorno afetivo bipolar $(14,4 \%)$ e episódios depressivos $(13,6 \%)$. Conclusão: o Centro de Atenção Psicossocial apresentou um percentual elevado de altas clínicas, garantindo o retorno dos usuários à vida cotidiana, especialmente entre os sujeitos com transtornos mentais graves e persistentes.

Descritores: Saúde mental; Serviços de saúde mental; Alta do paciente

\section{ABSTRACT}

Objective: characterize the discharge process of the users from a Psychosocial Care Center. Method: documentary study with a quantitative approach with data from the discharge record of users, from 2006 to 2016. The variables gender, reason for discharge and diagnosis were analyzed. Results: the study sample consisted of 1,772 discharges, distributed without great disparity between female (50.9\%) and male (46.1\%). The clinical discharge had the high rate (55.6\%) in the 11 years studied and among the three main diagnoses: bipolar affective disorder (62.1\%), episodes of depression

1 Enfermeira. Doutora em Enfermagem. Universidade Federal de Pelotas (UFPEL). Email: kantorski@uol.com.br http://orcid.org/0000-0001-9726-3162

2 Enfermeira. Doutora em Enfermagem. Universidade Federal de Pelotas (UFPEL). Email: arianecguedes@gmail.com http://orcid.org/0000-0002-5269-787X

3 Enfermeira, Mestre em Ciências. Instituto Federal de Educação, Ciência e Tecnologia do Rio Grande do Sul (IFRS). E-mail: roberta.machado@riogrande.ifrs.edu.br http://orcid.org/0000-0002-9087-6457

4 Psicóloga. Mestre Ciências. Universidade Federal de Pelotas (UFPEL). Email: marialauradeoliveiracouto@gmail.com http://orcid.org/0000-0002-5103-3000

5 Bacharel em Direito, Mestre em Ciências. Universidade Federal de Pelotas (UFPEL). Email: vini_boldt@yahoo.com.br http://orcid.org/0000-0001-7067-7920

6 Enfermeiro. Mestre em Ensino na Saúde. Universidade Federal de Santa Maria (UFSM). E-mail: guilhermeewp@politecnico.ufsm.br http://orcid.org/0000-0003-0069-7023

* Este artigo recebeu financiamento via Edital MCT-CNPq/MS-SCIETIE-DECIT/CT-Saúde-N 07/2005 e Edital CNPq $14 / 2012$. 


\section{JOURNALOF

(59.2\%) and schizophrenia (58.3\%). Schizophrenia was the prevalent diagnosis among users (24.2\%), after bipolar affective disorder (14.4\%), followed by depressive episodes (13.6\%). Conclusion: the Psychosocial Care Center presented a high percentage of clinical discharge, guaranteeing users' return to daily life, especially among subjects with severe and persistent mental disorders.

Descriptors: Mental health, Mental health services, Patient discharge

\section{RESUMEN}

Objetivo: caracterizar el alta de los usuarios en el Centro de Atención Psicosocial. Método: estudio documental con abordaje cuantitativo con datos del libro de registro de alta de los usuarios, de 2006 a 2016. Se analizaron las variables sexo, motivo de alta y diagnóstico. Resultados: la muestra constituyó 1772 altas, distribuidas sin gran disparidad entre el sexo femenino (50,9\%) y masculino $(46,1 \%)$. La alta clínica es el motivo prevalente $(55,6 \%)$ en los once años estudiados y entre los tres principales diagnósticos: trastorno afectivo bipolar (62,1\%), episodios de depresión $(59,2 \%)$ y esquizofrenia (58,3\%). La esquizofrenia fue el diagnóstico prevalente entre los usuarios $(24,2 \%)$, tras el trastorno afectivo bipolar (14,4\%), seguido de los episodios depresivos (13,6\%). Conclusión: el Centro de Atención Psicosocial presentó un porcentaje elevado de altas clínicas, garantizando el retorno de los usuarios a la vida cotidiana, especialmente entre los sujetos con trastornos mentales graves y persistentes.

Descriptores: Salud mental; Servicios de salud mental; Alta del paciente

\section{INTRODUÇÃO}

Os transtornos mentais são responsáveis por mais de um terço do número total de incapacidades nas Américas, atingindo pessoas de todas as faixas etárias. Os transtornos depressivos são a principal causa de doença e incapacidade seguido pelos transtornos de ansiedade. ${ }^{1}$ Houve um avanço na maioria dos países em direção à implantação de serviços de saúde mental, apesar de quase todos enfrentarem barreiras de consolidação de práticas fora do asilo, deixando grande parte das pessoas sem tratamento qualificado. ${ }^{1-3}$

Considerando o avanço da rede de atenção psicossocial e das práticas de cuidado no contexto brasileiro, que representaram a possibilidade de cuidado, de vida e liberdade para muitas pessoas, algumas barreiras precisam ser enfrentadas. Dentre elas, o desafio de problematizar a alta, ou uma categoria equivalente a ser pensada em serviços como os Centros de Atenção Psicossocial (CAPS).

Apesar da temática da alta dos usuários de serviços de saúde mental ser recente, ela tem sido amplamente discutida por pesquisadores de diversos países, tais como o Japão, que tem o maior número de leitos psiquiátricos $\mathrm{e}$ os seus hospitais têm a tendência de fornecer tratamento contínuo para os pacientes, ocasionando a longa permanência dos usuários nestes serviços. $^{2}$

Frente a isso, o Ministério da Saúde, Trabalho e Bem-estar do país anunciou uma política para a transição dos pacientes internados em hospitais psiquiátricos para serviços de saúde mental comunitários. Contudo, essa medida não foi satisfatória, pois os serviços comunitários do Japão apresentam um percentual de alta um pouco menor do que o dos hospitais, 


\section{JOURNALOF

fazendo com que o problema da institucionalização dos usuários apenas mude de lugar. $^{2}$

$\mathrm{Na}$ Inglaterra, uma pesquisa a fim de descrever as características clínicas e demográficas de todos os pacientes internados em um serviço de saúde mental urbano com atraso de alta de três meses, evidenciou que a demora na liberação da alta desses usuários era semelhante à de um grupo de comparação de longa permanência, constatando mais uma vez a necessidade de se trabalhar a questão da alta no intuito de que ela possa ser realizada com êxito. ${ }^{3-4}$ Esse serviço de saúde mental urbano, ao contrário dos CAPS tipo II, possui leitos psiquiátricos, caracterizando-se mais como um hospital do que um serviço substitutivo ao modelo manicomial.

Mesmo na Itália, um país onde não existem mais hospitais psiquiátricos desde a aprovação da Lei 180/1978, uma pesquisa realizada em todo o país, com o objetivo de avaliar a quantidade e a qualidade dos serviços, mostrou que em 1999 mais de um terço $(37,7 \%)$ das unidades de saúde mental comunitárias não ofereceram a alta para nenhum paciente e que $31,5 \%$ dos serviços liberaram apenas para um ou dois usuários, apontando o baixo índice de alta. ${ }^{3,5}$

Tendo em vista a importância do CAPS em proporcionar estratégias de "alta" para seus usuários e a existência de poucos estudos nacionais $e$ internacionais com o enfoque no tema em questão, este artigo objetiva caracterizar a alta dos usuários no CAPS.

\section{MATERIAIS E MÉTODOS}

Este estudo é do tipo documental, descritivo de abordagem quantitativa. Trata-se de um recorte da pesquisa "Avaliação dos Centros de Atenção Psicossocial da região sul do Brasil" (CAPSUL I e CAPSUL II), realizada em duas edições (2006 e 2016), realizando etapas quantitativas e qualitativas. $\mathrm{Na}$ primeira etapa quantitativa, realizada em 2006, foram avaliados 30 serviços em diferentes cidades em relação à estrutura, processo e resultado da atenção em saúde mental; e uma fase qualitativa em que foram selecionados cinco casos, tendo como parâmetro os dados obtidos na etapa de avaliação quantitativa, referentes à estrutura, processo e sua adequação às normas definidas pela Portaria do Ministério da Saúde $\mathrm{n}^{\circ} 336 / 2002,{ }^{6}$ o tempo de funcionamento e a experiência do serviço. Dentre os municípios estudados, apenas um serviço trabalhava explicitamente com a alta como um eixo para diminuir o tempo de permanência no serviço e potencializar a inserção no território, sendo este o caso tratado neste artigo e acompanhado ao longo de onze anos. 0 caso selecionado atende aos requisitos de acessibilidade das premissas, pertinência e suficiência, constituindo-se num caso qualificado para o estudo da temática. ${ }^{7}$

O local de estudo foi um Centro de Atenção Psicossocial localizado em uma cidade com a população estimada em 570.000 habitantes $^{8}$ e uma rede de atenção psicossocial articulada, permitindo o fluxo do usuário de saúde mental pelos serviços e instituições, sem hospital psiquiátrico na rede. 


\section{JOURNALOF \\ NURSING \\ ANO HEALTH}

ISSN 2236 - 1987

0 estudo respeitou as exigências formais contidas nas normas nacionais e internacionais regulamentadoras de pesquisas envolvendo seres humanos. A primeira edição foi apreciada pelo Comitê de Ética e Pesquisa da Faculdade de Medicina da Universidade Federal de Pelotas, recebendo parecer favorável pelo ofício $\mathrm{N}^{\circ} 074 / 05$ de 11 de novembro de 2005, enquanto que a segunda foi apreciada pelo Comitê de Ética e Pesquisa da Faculdade de Enfermagem da Universidade Federal de Pelotas, recebendo parecer favorável sob $N^{\circ} 176 / 2011$ de 21 de março de 2011.

\section{RESULTADOS}

A amostra se constituiu em 1772 usuários em alta no período dos onze anos estudados (2006 a 2016), constata-se na Tabela 1 uma aproximação entre 0 número de usuários referente ao sexo feminino (51\%) e masculino (46\%).

Com relação aos motivos que geraram a alta dos usuários do serviço (Tabela 1), a alta clínica é a causa mais prevalente nos onze anos estudados, responsável por mais da metade destas $(55,6 \%)$, seguida de alta por abandono $(29,6 \%)$, enquanto os outros cinco motivos abordados somados totalizam $14,8 \%$, os quais, mesmo somados à alta por abandono, não ultrapassam a alta clínica, totalizando $44,4 \%$ de altas. De 2010 a 2013 teve-se a maior taxa de alta clínica $(64,6 \%)$. A alta a pedido representa 7,9\% do total de liberações. 
Tabela 1: Sexo e motivos de alta em usuários de saúde mental

\begin{tabular}{|c|c|c|c|}
\hline \multirow[t]{2}{*}{ Variáveis } & 2006-2009 & $2010-2013$ & 2014-2016 \\
\hline & n (\%) & $\mathrm{n}(\%)$ & $\mathrm{n}(\%)$ \\
\hline \multicolumn{4}{|l|}{ Sexo } \\
\hline Feminino & $266(45,6)$ & $291(51,2)$ & $345(55,6)$ \\
\hline Masculino & $282(48,4)$ & $260(45,8)$ & $275(44,3)$ \\
\hline Não Informado & $35(6,0)$ & $17(3,0)$ & $1(0,2)$ \\
\hline Motivos & 1772 & 124 & \\
\hline Abandono & $116(19,9)$ & $121(21,3)$ & $215(34,6)$ \\
\hline Alta Clínica & $325(55,7)$ & $367(64,6)$ & $291(46,9)$ \\
\hline Encaminhamento & $14(2,4)$ & $13(2,3)$ & $49(7,9)$ \\
\hline Mudança de Endereço & $0(0,0)$ & $13(2,3)$ & $14(2,3)$ \\
\hline Não Informado & $62(10,6)$ & $9(1,6)$ & $5(0,8)$ \\
\hline Óbito & $8(1,4)$ & $3(0,5)$ & $7(1,1)$ \\
\hline Pedido & $58(9,9)$ & $42(7,4)$ & $40(6,4)$ \\
\hline Total $(n=1772)$ & $583(100,0)$ & $568(100,0)$ & $621(100,0)$ \\
\hline
\end{tabular}

Fonte: dados da pesquisa, 2018

Quanto aos diagnósticos mais recorrentes (Tabela 2), a esquizofrenia foi responsável por $24,2 \%$ do total dos casos, sendo os anos de 2006 a 2009, os que obtiveram maior prevalência (34,3\%). O transtorno afetivo bipolar ficou em segundo lugar $(14,4 \%)$, dos diagnósticos, ocorrendo maior prevalência nos anos de 2014 a 2016 (16,1\%). E os episódios depressivos ficaram em terceiro lugar $(13,6 \%)$, alcançando a maior prevalência $(16,6 \%)$ nos anos de 2010 a 2013.

Ao cruzar diagnósticos de alta com os motivos, foi possível verificar que, nos onze anos estudados 66,7\% dos usuários com transtornos esquizotípicos e delirantes possuem como principal motivo a alta clínica. Entre as pessoas com diagnósticos de transtorno afetivo bipolar, a alta clínica ocorreu em $62,1 \%$ dos casos, com diagnóstico de depressão 59,2\%, e esquizofrenia $58,3 \%$.

Os registros em livros de admissão datavam apenas os anos de 2006 e 2007 sobre o tempo de permanência do usuário no serviço. Evidenciou-se que $45,5 \%$ dos usuários deste serviço apresentavam um tempo médio de permanência de 6 a 12 meses. Em 2006, a maioria dos usuários ficavam 159 dias em média em tratamento e 22 dias no ano de 2007 , somando ambos os anos, a média foi de 87 dias. 
Tabela 2. Diagnósticos dos usuários de saúde mental

\begin{tabular}{|c|c|c|c|}
\hline \multirow[t]{2}{*}{ Variáveis } & $2006-2009$ & $2010-2013$ & $2014-2016$ \\
\hline & $\mathrm{n}(\%)$ & $\mathrm{n}(\%)$ & $\mathrm{n}(\%)$ \\
\hline Episódio Maníaco & $6(1,0)$ & $3(0,5)$ & $1(0,2)$ \\
\hline Episódios Depressivos & $78(13,4)$ & $94(16,6)$ & $69(11,1)$ \\
\hline Esquizofrenia & $200(34,3)$ & $118(20,8)$ & $111(17,8)$ \\
\hline $\begin{array}{l}\text { Outros Transtornos Ansiosos } \\
\text { Transt. Ment. devidos à Lesão e Disf. }\end{array}$ & $14(2,4)$ & $23(4,1)$ & $11(1,8)$ \\
\hline Cerebral e a Doença Física & $0(0,0)$ & $1(0,2)$ & $11(1,8)$ \\
\hline $\begin{array}{l}\text { Psicose Não-orgânica Não Especificada } \\
\text { Reações ao "stress"' Grave e Transtornos de }\end{array}$ & $2(0,3)$ & $13(2,3)$ & $28(4,5)$ \\
\hline Adaptação" & $3(0,5)$ & $4(0,7)$ & $4(0,6)$ \\
\hline Retardo Mental Leve & $3(0,5)$ & $9(1,6)$ & $9(1,4)$ \\
\hline Transtorno Afetivo Bipolar & $70(12,0)$ & $85(15,0)$ & $100(16,1)$ \\
\hline $\begin{array}{l}\text { Transtorno Depressivo Recorrente } \\
\text { Transtorno Esquizotípico e Transtorno }\end{array}$ & $56(9,6)$ & $39(6.9)$ & $54(8,7)$ \\
\hline Obsessivo Compulsivo & $4(0,7)$ & $2(0,4)$ & $0(0,0)$ \\
\hline Transtornos Dissociativos (de Conversão) & $5(0,9)$ & $4(0,7)$ & $3(0,5)$ \\
\hline Transtornos Específicos da Personalidade & $14(2,4)$ & $29(5,1)$ & $10(1,6)$ \\
\hline $\begin{array}{l}\text { Transtornos Esquizoafetivos } \\
\text { Transtornos Mentais e Comportamentais }\end{array}$ & $42(7,2)$ & $23(4,1)$ & $16(2,6)$ \\
\hline $\begin{array}{l}\text { Devidos ao Uso de Álcool } \\
\text { Transtorno Devido ao Uso de Múlti. Drogas e }\end{array}$ & $6(1,0)$ & $5(0,9)$ & $4(0,6)$ \\
\hline Outras Substâncias & $2(0,3)$ & $3(0,5)$ & $5(0,8)$ \\
\hline Transtornos Psicóticos Agudos e Transitórios & $5(0,9)$ & $4(0,7)$ & $6(1,0)$ \\
\hline Outros & $9(1,5)$ & $21(3,7)$ & $16(2,6)$ \\
\hline Não Informado & $64(11,0)$ & $86(15,2)$ & $165(26,5)$ \\
\hline Total $(n=1.772)$ & $583(100,0)$ & $566(100,0)$ & $623(100,0)$ \\
\hline
\end{tabular}

Fonte: dados da pesquisa, 2018

\section{DISCUSSÃO}

A partir da caracterização da alta, o estudo mostra a sua aplicabilidade prática no fato de o Centro de Atenção Psicossocial se constituir como um dispositivo de cuidado transitório, empenhando-se na alta clínica, na diminuição do tempo de permanência e na inserção no território.
Com relação aos resultados, diferentemente de outras pesquisas desenvolvidas na área da saúde, notouse a predominância de usuários do sexo masculino. ${ }^{9-10}$

A alta clínica foi o motivo mais prevalente entre os usuários estudados, o que consiste num resultado da qualidade do serviço ofertado. Procurar determinar se o serviço produz o impacto que se espera na saúde dos usuários é um critério 


\section{JOURNALOF \\ NURSING \\ AND HEALTH}

ISSN 2236 - 1987

cinco motivos de alta
(encaminhamentos, mudança de endereço, não informados, óbitos e pedidos), ainda assim este número foi inferior ao motivo de alta clínica, o que demonstrou a qualidade do serviço prestado. A alta por abandono, com todas as suas possibilidades e justificativas para acontecer, como abuso de álcool e outras drogas, dificuldades de acessar o serviço ou a não adaptação ao tratamento, como verificamos ocorrer frequentemente em outros serviços, resultou em um baixo índice de ocorrências no presente estudo. ${ }^{14}$

A alta a pedido representou 7,9\%, sendo superior às altas por óbito, encaminhamentos e não informados. Enquanto a alta clínica sugere um cuidado no território e um acompanhamento, o pedido de alta pode significar um distanciamento do serviço.

Evidencia-se ainda que algumas variáveis de diagnóstico e tratamento podem ser mais preditoras de abandono, como os transtornos de humor e de ansiedade. ${ }^{15}$ Nesse sentido, a elevada prevalência de diagnóstico de oscilações de humor obtida nos dados deste estudo pode estar relacionada à alta por abandono.

Com relação aos diagnósticos e a prevalência das altas, a esquizofrenia foi 0 transtorno mais recorrente (24,2\%). A Organização Mundial da Saúde ${ }^{16-17}$ evidencia que a esquizofrenia atinge 21 milhões de pessoas em todo o mundo, sendo mais prevalente entre os homens (12 milhões) do que nas mulheres $(9$ milhões), dado que converge com os resultados obtidos. 


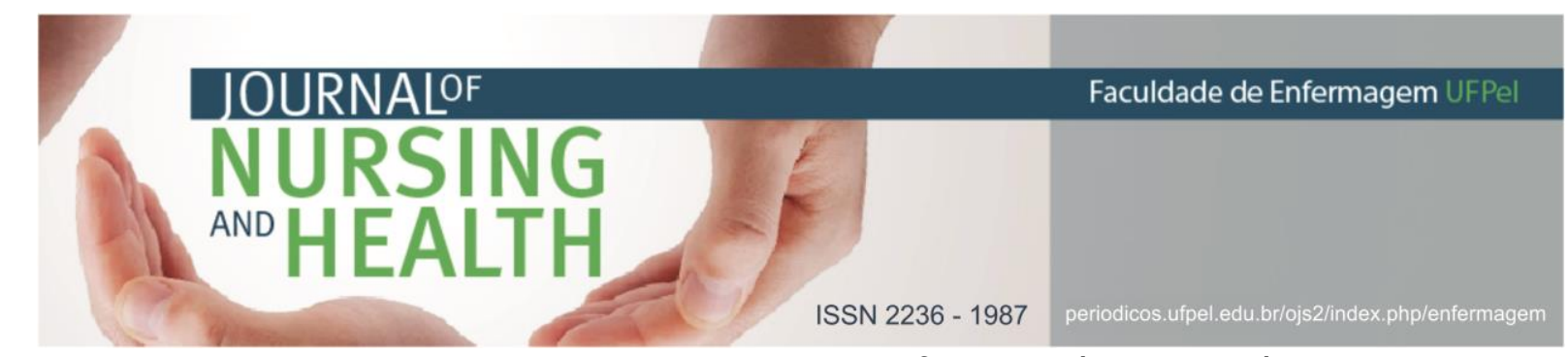

Entre os transtornos psiquiátricos, vem ocorrendo um aumento na prevalência dos transtornos de humor, ${ }^{17}$ sendo que, neste estudo, o transtorno bipolar encontra-se em segundo lugar $(14,4 \%)$ entre os diagnósticos dos usuários com alta, seguido dos episódios depressivos $(13,6 \%)$.

Uma pesquisa verificou no Brasil uma prevalência de diagnósticos de depressão em adultos de $7,6 \%$, sendo maior em mulheres $(10,9 \%)$ e em pessoas entre 60 e 64 anos, ${ }^{18}$ que corrobora com os dados encontrados na presente pesquisa, cuja incidência do sexo feminino com depressão é $34,3 \%$ maior do que em homens.

\section{CONSIDERAÇÕES FINAIS}

O Centro de Atenção Psicossocial apresentou um percentual elevado de altas clínicas, garantindo o retorno dos usuários à vida cotidiana, especialmente entre os sujeitos com transtornos mentais graves e persistentes.

Os resultados obtidos apresentaram como limite a escassez de registros detalhados sobre a alta no serviço, considerando que mesmo que a alta tenha sido estudada ao longo de onze anos, as variáveis disponíveis referiam-se apenas ao sexo, motivo e diagnóstico, enquanto havia registro quanto ao tempo de permanência do usuário no serviço em apenas dois anos neste período.

Outro limite consiste em tratar-se de um estudo descritivo considerando a limitação das variáveis disponíveis. Entretanto, é um estudo importante para a área da saúde mental, pois tem a função de estimular pesquisas e práticas de alta nos Centros de Atenção Psicossocial, construindo estratégias de reabilitação psicossocial e reinserção social dos sujeitos em sofrimento psíquico.

Assim, são necessários aprofundamentos e novos estudos sobre a alta nos serviços de atenção psicossocial, além da necessidade de uma análise aprofundada sobre a permanência dos usuários e os motivos das altas nestes serviços.

\section{REFERÊNCIAS}

1 Organización Panamericana de la Salud (OPS). La carga de los trastornos mentales en la región de las américas [Internet]. Washington DC; 2018[citado 2019 jun 14]. Disponible en: http://iris.paho.org/xmlui/bitstream/ handle/123456789/49578/9789275320 280_spa.pdf?sequence=9\&isAllowed=y

2 Tanioka T, Chiba S, Onishi Y, Kataoka $M$, Kawamura A, Tomotake $M$, et al. Factors associated with discharge of long-term inpatients with schizophrenia in japan: a retrospective study. Issues Ment Health Nurs [Internet]. 2013 Apr[cited 2019 Jun 14];34(4):256-64. Available from: https: / / www.tandfonline.com/doi/ful l/10.3109/01612840.2012.742602

3 de Girolamo G, Candini V, Buizza C, Ferrari C, Boero ME, Giobbio GM, et al. Is psychiatric residential facility discharge possible and predictable? A multivariate analytical approach applied to a prospective study in Italy. Soc Psychiatry Psychiatr Epidemiol[Internet]. 2014 Jan[cited 2019 Jun 14];49(1):157-67. Available from: 
https: / /link.springer.com/article/10. 1007\%2Fs00127-013-0705-z

4 Poole R, Pearsall A, Ryan T. Delayed discharges in an urban in-patient mental health service in England. Psychiatr Bull [Internet]. 2014 Apr[cited 2019 Apr 07];38(2):66-70. Available from: https://www.ncbi.nlm.nih.gov/pmc/a rticles/PMC4115402/pdf/pbrcpsych_3 8_2_005.pdf

5 Guedes AC, Olschowsky A, Kantorski LP, Antonacci MH. Violence social representations and teaching strategies used by undergraduate nursing professors. Rev. eletrônica enferm. [Internet]. 2017[cited 2019Apr 07];19(2017):1-9. Available from: https: / / revistas.ufg.br/fen/article/vi ew/43794/24695

6 Ministério da Saúde (BR). Legislação em saúde mental: 1990-2004 [Internet]. Brasília; 2004[acesso em 2019abr 07]. Disponível em: http://bvsms.saude.gov.br/bvs/public acoes/legislacao_saude_mental_1990_ 2004_5ed.pdf

7 Cardano M. Manual de pesquisa qualitativa: a contribuição da teoria da argumentação. Rio de Janeiro: Vozes; 2017.

8 Instituto Brasileiro de Geografia e Estatística (IBGE). Joinvile panorama [Internet]. 2017[acesso 2019 abr 07]. Disponível em: https://cidades.ibge.gov.br/brasil/sc /joinville/panorama

9 Carlotto MS, Barcinski M, Fonseca R. Transtornos mentais comuns $\mathrm{e}$ associação com variáveis sociodemográficas e estressores ocupacionais: uma análise de gênero. Estud. pesqui. psicol. (Impr.).
[Internet]. 2015[acesso em $2019 \mathrm{abr}$ 07]; 15(3):1006-26. Disponível em: http://pepsic.bvsalud.org/pdf/epp/v 15n3/v15n3a13.pdf

10 Senicato C, Azevedo RS, Barros MA. Transtorno mental comum em mulheres adultas: identificando os segmentos mais vulneráveis. Ciênc. Saúde Colet. [Internet]. 2018 Ago[acesso em 2019 mai 17];23(8):2543-54. Disponível em: http://www.scielo.br/scielo.php?scrip $\mathrm{t}=\mathrm{sci}$ arttext\&pid=S1413812320180008 02543\&lng=pt.

11 Costa PHA, Colugnati FB, Ronzani Telmo M. Avaliação de serviços em saúde mental no Brasil: revisão sistemática da literatura. Ciênc. Saúde Colet. [Internet]. 2015[acesso em 2019 mai 17];20(10):3243-53. Disponível em:

http:/ /www.scielo.br/scielo.php?scrip $\mathrm{t}=\mathrm{sci}$ _arttext\&pid=S1413812320150010 03243\&lng=en.

12 McMinn BG, Lewin TJ, Savio N, Matters D, Smith C. Readiness for discharge from subacute mental health units for older people: using available measures to support clinical decisionmaking. Int J Ment Health Nurs [Internet]. 2017[cited 2019 Apr 07];26(2):151-9. Available from: https://onlinelibrary.wiley.com/doi/e pdf/10.1111/inm.12282

13 Fortes FLS, Peres MAA, Santos TCF, Martins GCS, Montenegro HRA, Almeida Filho AJ. Mental health nurses: conceptions about professional qualification in a psychosocial care center. Rev Rene (Online). [Internet]. 2017[cited 2019 Apr 07]; 18(6):76370. Available 


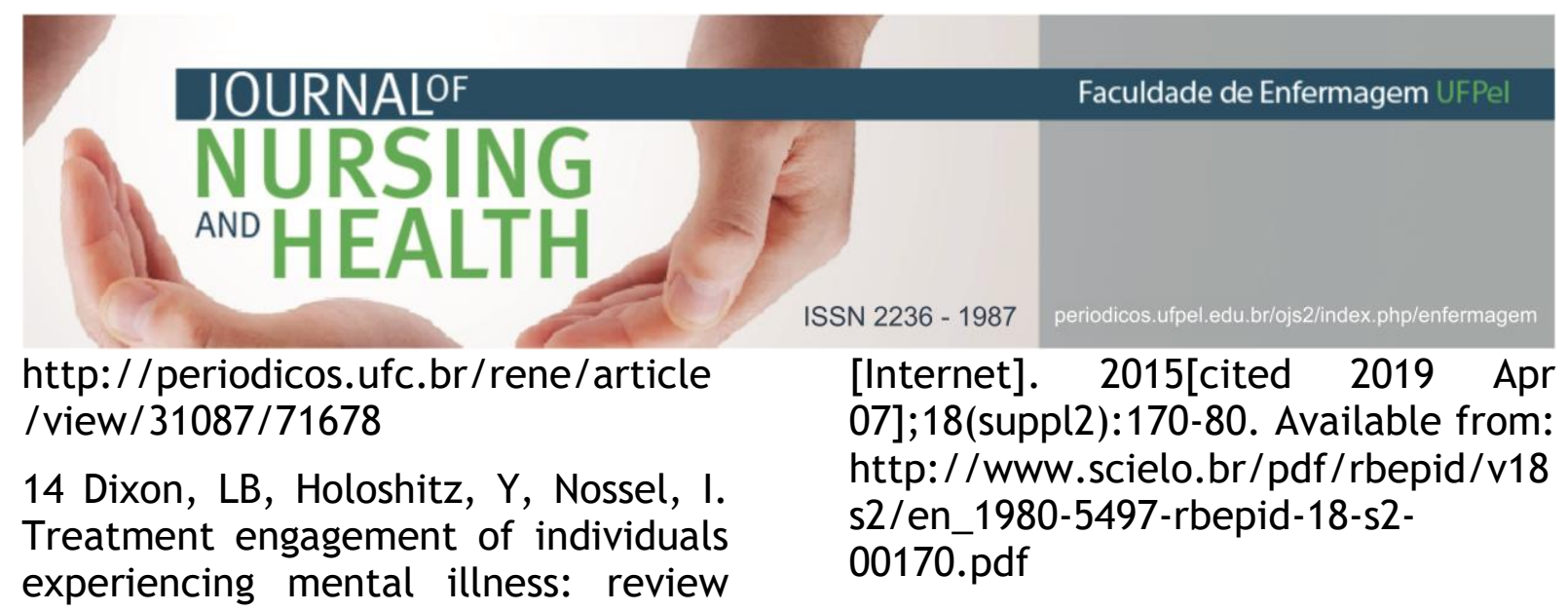

and update. World psychiatry [Internet]. 2016 Feb[cited 2019 Jun 14];15(1):13-20. Available from: https://www.ncbi.nlm.nih.gov/pmc/a rticles/PMC4780300/

Data de submissão: 02/04/2019

Data de aceite: 24/05/2019

Data de publicação: 18/06/2019

15 Organización Mundial de la Salud (OMS). Nota descritiva $N^{\circ} 397$ : esquizofrenia [Internet]. 2016[citado abr 07]. Disponible en: http://www.who.int/mediacentre/fa ctsheets/fs397/es/

16 Jongsma HE, Gayer-Anderson C, Lasalvia A, Quattrone D, Mulè A, Szöke $A$, et al. Treated incidence of psychotic disorders in the multinational EU-GEI study. JAMA Psychiatry [Internet]. 2017[cited 2019 Apr 07];75(1):36-46. Available from: https://www.ncbi.nlm.nih.gov/pmc/a rticles/PMC5833538/

17 Arrais PSD, Fernandes MEP, Pizzol TSD, Ramos LR, Mengue SS, Luiza VL, et al. Prevalence of self-medication in Brazil and associated factors. Rev. saúde pública (Online). [Internet]. 2016[cited 2019 Apr 08];50(suppl2):13s. Available from:http://www.rsp.fsp.usp.br/wpcontent/uploads/articles_xml/00348910-rsp-s2-S01518-

87872016050006117/0034-8910-rsp-s2S01518-

87872016050006117.x83745.pdf

18 Stopa SR, Malta DC, Oliveira MM, Lopes CS, Menezes PR, Kinoshita RT. Prevalence of self-reported depression in Brazil: 2013 national health survey results. Rev. bras. epidemiol. 